\title{
The Implication of Organelle Cross Talk in AKI
}

\author{
Reiko Inagia, b \\ aDivision of CKD Pathophysiology, The University of Tokyo Graduate School of Medicine, Tokyo, Japan; \\ ${ }^{b}$ Division of Nephrology and Endocrinology, The University of Tokyo Graduate School of Medicine, Tokyo, Japan
}

\begin{abstract}
Keywords
Endoplasmic reticulum stress - Mitochondrial stress . Unfolded protein response - Endoplasmic reticulummitochondrial interaction - Fatty acid metabolism . Lipotoxicity $\cdot$ Tubular inflammation
\end{abstract}

\begin{abstract}
Organelle stress, such as mitochondrial or endoplasmic reticulum damage, plays a crucial role in the pathogenesis of acute kidney injury (AKI). Further, persistent organelle stress, which causes metabolic abnormality followed by inflammation and fibrosis, is an important mediator of AKI-to-CKD transition. Organelle stress closely links to the derangement of organelle cross talk. Organelles intricately interact with each other under the physiological conditions to maintain their function each other and subsequent cell fate. Organelle stress and their cross talk are now a focus of intensive researches in the field of AKI as they are in the field of CKD.

\footnotetext{
(C) 2020 The Author(s)

Published by S. Karger AG, Basel
}

\section{Introduction}

When the kidney function declines under the pathogenic conditions, such as genetic abnormalities or unfavorable lifestyle, endoplasmic reticulum (ER) or mitochondrial damages, referred to as organelle stress, in var-

\section{karger@karger.com www.karger.com/nef

ious kidney cells are observed as kidney disease progresses $[1,2]$. The ER regulates protein quality control, and mitochondria synthesize ATP through the tricarboxylic acid cycle and oxidative phosphorylation (OXPHOS). Under the physiological conditions, ER or mitochondrial stress induces adaptive stress signals to maintain cellular homeostasis. However, pathogenic and maladaptive organelle stress induces various phenotypic changes of tubules in acute kidney injury (AKI), including tubular cell death and inflammation. Further, it has become a consensus that organelle stress does not occur in each organelle independently. For example, ER interacts with mitochondria for maintaining the organelle function of each other [3]. The detective ER-mitochondria interaction contributes to disease progression in association with organelle damage $[4,5]$. The pathophysiological and therapeutic impacts of organelle stress and organelle cross talk are, thus, new research filed in AKI and CKD (Fig. 1).

Contribution from the AKI and CRRT 2020 Symposium at the 25th International Conference on Advances in Critical Care Nephrology, Manchester Grand Hyatt, San Diego, CA, USA, February 24-27, 2020. This symposium was supported in part by the NIDDK funded University of Alabama at Birmingham-University of California San Diego O'Brien Center for Acute Kidney Injury Research (P30DK079337). 
Fig. 1. ER-mitochondrial cross talk in AKI and AKI-to-CKD transition. ER and mitochondria are organelles acting for protein homeostasis and energy metabolism, respectively, and interact for maintaining the functions of each other, for example, by MAM. In contrast, ER stress or mitochondria stress as well as damaged ER-mitochondrial cross talk causes tubular damage. This leads to lipid metabolic abnormality, inflammation, and fibrosis in AKI and AKI-to-CKD transition (Ref. [17] with modifications). AKI, acute kidney injury; $\mathrm{ER}$, endoplasmic reticulum; MAM, mitochondria-associated ER membranes.

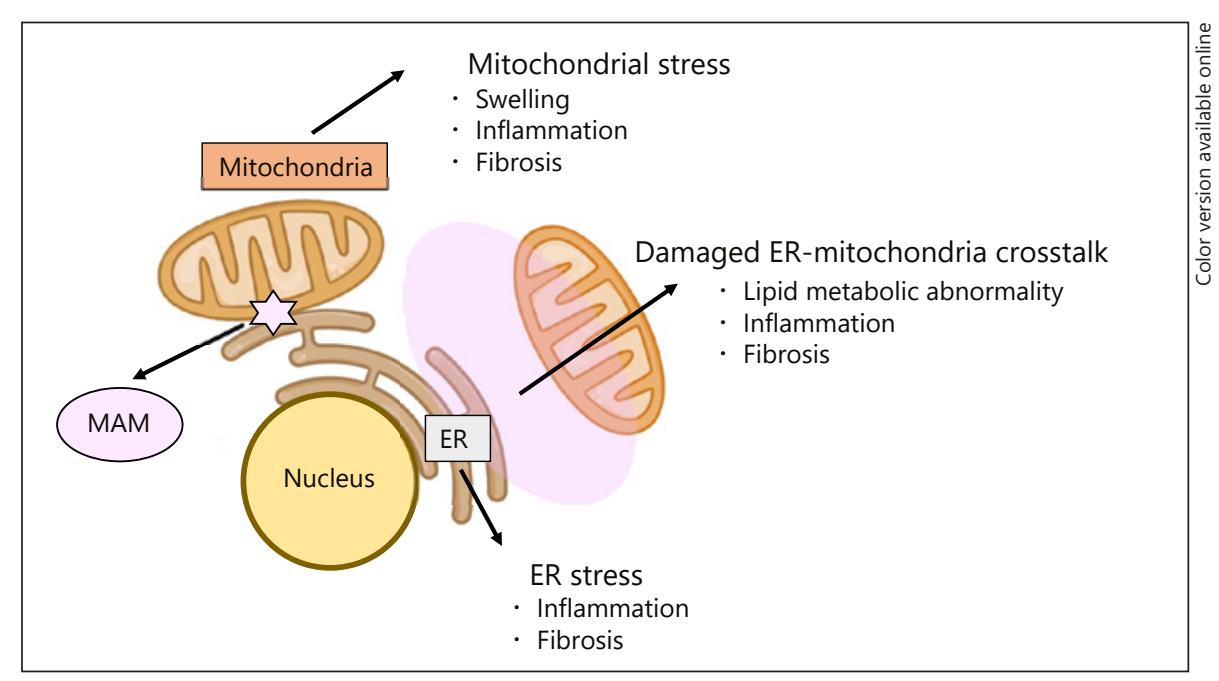

\section{ER Function Is Critical for Cellular Homeostasis}

Physiological ER stress activates the unfolded protein response (UPR) pathway, which is regulated by the 3 major transducers in the ER lumen such as inositol-requiring protein 1 , pancreatic eukaryotic translation initiation factor $2 \alpha$ kinase, and activating transcription factor 6 (ATF6) [5]. The transducers sense the decline of ER function by dissociation from glucose-regulated protein 78 (GRP78)/binding immunoglobulin protein (BiP) and activate the downstream UPR transcription factors of spliced Xbox-binding protein 1 (sXBP1) and ATF4. This process upregulates the expression of UPR target genes involved in protein folding and ER-associated protein degradation. Meanwhile, activated ATF6 by dissociation from GRP78/BiP translocates into the Golgi apparatus, where it is cleaved to give rise to ATF6 (p50), a transcription factor, which also activates the expression of the UPR target genes. ER stress also induces UPR-related apoptosis, which occurs via the activation of CCAAT/enhancer-binding protein homologous protein. Physiological ER stress induces adaptive UPR pathway for ER and subsequent homeostasis. In contrast, AKI is associated with pathogenic ER stress in damaged tubular cells, which subsequently induces maladaptive UPR pathway and contributes to the progression of AKI.

\section{Mitochondrial Function Is Crucial for Cellular Energy Metabolism}

Mitochondria are essential for ATP production through OXPHOS. OXPHOS complexes I-V are located in the mitochondrial inner membrane [2]. During OX-
PHOS, electrons are transferred from electron donors to electron acceptors such as oxygen in redox reactions. These redox reactions release the energy stored in the relatively weak double bond of $\mathrm{O}_{2}$, which is used to form ATP. Mitochondria fulfill the high metabolic energy demands of the kidney, and especially proximal tubules are enriched with mitochondria. Mitochondrial dysfunction in AKI results in not only the shortage of ATP production but also oxidative stress [2]. The free radical attack occurs directly at complexes in the mitochondrial respiratory chain. Complexes I and III are also thought to be major sites for the production of superoxide and other reactive oxygen species. Mitochondrial dysfunction also results in a release of pro-apoptotic factors. In ischemic conditions, the mitochondrial permeability transition pore, a nonspecific pore in the inner mitochondrial membrane, opens and causes irreversible damage.

\section{Organelle Stress Contributes to the Progression of AKI}

Recently accumulating evidence demonstrates the molecular mechanisms of how ER or mitochondrial dysfunction implicates tubular damage in AKI and highlights the impact of the pathophysiology of organelle stress, such as ER stress or mitochondrial stress.

\section{ER Stress}

In tubular damage in AKI, ER stress is closely associated with various phenotypic changes in tubular cells. For example, an updated report demonstrates that increased sXBP1-mediated signaling in tubules is unique to sepsis- 
induced AKI and contributes to renal inflammation and injury [6]. Using the cultured kidney, tubular cell model of hypoxia reoxygenation (oxidative stress) and ER stress, we found that the expression of miR-205 was markedly decreased in association with tubular cell death in both stress conditions. MiR-205 bound to the $3^{\prime}$-UTR of the prolyl hydroxylase 1 (PHD1/EGLN2) gene and suppressed the transcription level of EGLN2, which modulates both intracellular oxidative stress and ER stress state, serving a protective role against both oxidative and ER stresses in AKI [7]. Further, overwhelming ER stress is also induced in kidneys in the mouse model of AKI-toCKD transition after unilateral renal ischemia-reperfusion injury [8]. Importantly, 2 chemical chaperones, TUDCA and 4-PBA, blocked ER stress in postischemic kidneys and promoted renal recovery with suppression of renal fibrosis and myofibroblast activation.

\section{Mitochondrial Stress}

In AKI, damaged mitochondria release danger molecules, such as reactive oxygen species and mitochondrial DNA (mtDNA), which aggravates inflammation. Our group recently demonstrated that the cyclic GMP-AMP synthase (cGAS) stimulator of interferon genes (STING) pathway detects mtDNA released from damaged mitochondria and induces innate immunity in damaged tubular cells in AKI [9]. In cisplatin-induced AKI mice, cisplatin caused mtDNA leakage into the cytosol through BCL-2-like protein 4 (BAX) pores in the mitochondrial outer membrane in tubules, with subsequent activation of the cGAS-STING pathway, thereby triggering inflammation and AKI progression. We also used a genetically engineered mouse model of cisplatin-induced AKI and observed improvement of the disease by systemic STING deficiency. Our data are consistent with the article reported by Chung et al. [10]. They generated mice with tubulespecific deletion of the mitochondrial transcription factor A (TFAM). In tubular cells of TFAM knockout mice, aberrant packaging of the mtDNA resulted in its cytosolic translocation, activation of the cGAS-STING pathway followed by tubular inflammation.

Szeto and colleagues conducted a 9-month study in rats with bilateral renal ischemia [11]. They detected the progression of peritubular capillary rarefaction, macrophage infiltration, and fibrosis with mitochondrial degeneration and mitophagy over the study period. Treatment with a mitoprotective agent (SS-31; elamipretide) for 6 weeks, starting 1 month after ischemia, ameliorated these disease phenotypes.
Sepsis-associated AKI also shows the tubular mitochondrial impairment with their swelling [12]. PPAR $\gamma$ coactivator-1 $\alpha$ (PGC-1 $\alpha)$ is a major regulator of mitochondrial biogenesis and metabolism. Global and tubulespecific PGC-1 $\alpha$-knockout mice had a normal basal renal function but suffered a persistent injury following endotoxemia, demonstrating the importance of PGC-1 $\alpha$ for recovery of kidney function. Further, PGC1 $\alpha$ is a pivotal determinant of renal recovery from injury by regulating nicotinamide adenine dinucleotide (NAD) biosynthesis $[13,14]$. Pgcla-deficient mice with renal ischemia developed local deficiency of the NAD precursor niacinamide (also known as nicotinamide), marked fat accumulation, and failure to re-establish normal function, suggesting niacinamide and NAD as key effectors for achieving PGC1 $\alpha$-dependent stress resistance [14].

\section{Inter-Organelle Cross Talk Is the Key to Understand the Pathomechanism of Kidney Disease}

Further, recent technological advances revealed critical roles of the interaction of organelles for the maintenance of organelle homeostasis and the subsequent cell fate. It became possible to visualize the frequency and location of interactions among different organelles, such as ER, Golgi apparatus, lysosome, peroxisome, or mitochondria, and these investigators may provide new insights regarding the pathophysiological dynamics of these interactions in AKI. ER and mitochondria are highly interconnected with a specialized set of proteins in cells. ER-mitochondrial interconnections form specific microdomains, called mitochondria-associated ER membranes (MAM), which play important roles in calcium signaling, lipid homeostasis, mitochondrial dynamics, inflammation, and autophagy $[3,4]$. In kidney disease, mitochondria-ER cross talk may be deranged, and it thereby activates the pathogenic signals that induce tubular metabolic alteration, organelle-mediated inflammatory pathway, and profibrotic response, in turn leading to kidney dysfunction with AKI. Lipids made in the ER membrane can be sent to the Golgi via specialized transfer proteins, and specific phospholipid synthases are enriched at ER-mitochondria junctions to more efficiently expedite phospholipid transfer. As a hub for protein and lipid synthesis, the ER is an important cellular organelle. Our group showed that lipotoxicity plays a crucial role in the pathogenesis of kidney disease [15, 16], and a role of organelle cross talk in lipotoxicity, for example, regulation of mitochondrial $\beta$-oxidation by 
UPR pathway [16] is an important subject for future studies.

Until now, the evidence showing the role of MAM in tubular cells in AKI is not reported yet. However, as described above, cytosolic mtDNA activates cGAS-STINGmediated tubular inflammation in AKI [9]. From the viewpoint of that STING is an ER-resident membrane protein, the mtDNA-cGAS-STING pathway is one of the mechanisms of ER-mitochondria cross talk, which works independently of MAM.

\section{Conclusion}

Organelle stress, which is associated with mitochondrial or ER dysfunction, mediates tubular inflammation and fibrosis, playing a crucial role in the pathogenesis of kidney disease, including AKI. Organelle stress closely links to the derangement of organelle cross talk, which is a focus of intensive researches in the field of nephrology. Understanding of organelle stress followed by deranged organelle cross talk is crucial to understand the pathophysiology of kidney disease.

\section{Acknowledgment}

This work is supported by Grant-in-Aid for Scientific Research (B) (JSPS KAKENHI grant 18H02727) and the research grant from Kyowa Kirin Co. Ltd.

\section{Conflict of Interest Statement}

The author has no conflicts of interest to declare.

\section{References}

1 Maekawa H, Inagi R. Pathophysiological role of organelle stress/crosstalk in AKI-to-CKD transition. Semin Nephrol. 2019 Nov;39(6): 581-8.

2 Bhargava P, Schnellmann RG. Mitochondrial energetics in the kidney. Nat Rev Nephrol. 2017;13(10):629-46.

3 Missiroli S, Patergnani S, Caroccia N, Pedriali G, Perrone M, Previati M, et al. Mitochondria-associated membranes (MAMs) and inflammation. Cell Death Dis. 2018 Feb 28;9(3): 329.

4 Resende R, Fernandes T, Pereira AC, De Pascale J, Marques AP, Oliveira P, et al. Mitochondria, endoplasmic reticulum and innate immune dysfunction in mood disorders: do mitochondria-associated membranes (MAMs) play a role? Biochim Biophys Acta Mol Basis Dis. 2020 Jun 1;1866(6):165752.

5 Inoue $\mathrm{T}$, Maekawa $\mathrm{H}$, Inagi $\mathrm{R}$. Organelle crosstalk in the kidney. Kidney Int. 2019 Jun; 95(6):1318-25.

6 Ferrè S, Deng Y, Huen SC, Lu CY, Scherer PE, Igarashi $\mathrm{P}$, et al. Renal tubular cell spliced $\mathrm{X}$ box binding protein 1 (Xbp1s) has a unique role in sepsis-induced acute kidney injury and inflammation. Kidney Int. 2019 Dec;96(6): 1359-73.
7 Muratsu-Ikeda S, Nangaku M, Ikeda Y, Tanaka T, Wada T, Inagi R. Downregulation of miR-205 modulates cell susceptibility to oxidative and endoplasmic reticulum stresses in renal tubular cells. PLoS One. 2012;7(7): e41462.

8 Shu S, Zhu J, Liu Z, Tang C, Cai J, Dong Z. Endoplasmic reticulum stress is activated in post-ischemic kidneys to promote chronic kidney disease. EBioMedicine. 2018 Nov;37: 269-80.

9 Maekawa $\mathrm{H}$, Inoue $\mathrm{T}$, Ouchi $\mathrm{H}$, Jao TM, Inoue R, Nishi H, et al. Mitochondrial damage causes inflammation via cGAS-STING signaling in acute kidney injury. Cell Rep. 2019; 29(5):1261-e6.

10 Chung KW, Dhillon P, Huang S, Sheng X, Shrestha R, Qiu C, et al. Mitochondrial damage and activation of the STING pathway lead to renal inflammation and fibrosis. Cell Metab. 2019 Oct 1;30(4):784-e5.

11 Szeto HH, Liu S, Soong Y, Seshan SV, CohenGould L, Manichev V, et al. Mitochondria protection after acute ischemia prevents prolonged upregulation of IL-1. J Am Soc Nephrol. 2017 May;28(5):1437-49.
12 Tran M, Tam D, Bardia A, Bhasin M, Rowe GC, Kher A, et al. PGC-1 $\alpha$ promotes recovery after acute kidney injury during systemic inflammation in mice. J Clin Invest. 2011 Oct; 121(10):4003-14.

13 Tran MT, Zsengeller ZK, Berg AH, Khankin EV, Bhasin MK, Kim W, et al. PGC1a drives NAD biosynthesis linking oxidative metabolism to renal protection. Nature 2016 Mar 24; 531(7595):528-32.

14 Ralto KM, Rhee EP, Parikh SM. NAD+ homeostasis in renal health and disease. Nat Rev Nephrol. 2020 Feb;16(2):99-111.

15 Anusornvongchai T, Nangaku M, Jao TM, $\mathrm{Wu} \mathrm{CH}$, Ishimoto $\mathrm{Y}$, Maekawa $\mathrm{H}$, et al . Palmitate deranges erythropoietin production via transcription factor ATF4 activation of unfolded protein response. Kidney Int. 2018 Sep;94(3):536-50.

16 Jao TM, Nangaku M, Wu CH, Sugahara M, Saito H, Maekawa H, et al . ATF6a downregulation of PPARa promotes lipotoxicity-induced tubulointerstitial fibrosis. Kidney Int. 2019 Mar;95(3):577-89.

17 Hasegawa S, Inagi R. Organelle stress and crosstalk in kidney disease. Kidney 360. 2020. Forthcoming. 